\title{
Postoperative Bleeding Risk Under Continued the P2Y12 Receptor Antagonists Ticagrelor
}

\author{
TRAN Thanh Huyen ${ }^{1}$ MD, PHAM Thanh Dat $^{2,3}$ MD \\ ${ }^{1}$ Department of Cardiology, G. Montpied, Clermont-Ferrand University Hospital, France \\ ${ }^{2}$ Department of Cardiovascular surgery, G. Montpied, Clermont-Ferrand University Hospital, France \\ ${ }^{3}$ Cardiovascular Center - E Hospital, Hanoi, Vietnam
}

\begin{abstract}
Introduction: Ticagrelor is used for the prevention of thrombotic events in patients with acute coronary syndrome or myocardial infarction with ST elevation. The drug is combined with acetylsalicylic acid (ASA - Aspirin) unless the latter is contraindicated. The FDA indication for ticagrelor is reduction of the rate of cardiovascular death, myocardial infarction (MI), and stroke in people with acute coronary syndrome or history of myocardial infarction. AHA/ACC Guidelines 2014 state: It is reasonable to choose ticagrelor over clopidogrel for P2Y12 inhibition treatment in patients with NSTE-ACS treated with early invasive strategy and/or coronary stenting [1]. The most common side effects are shortness of breath (dyspnea, 14\%) and various types of bleeding, such as hematoma, gastrointestinal, subcutaneous, intracranial hemorrhage... In addition to this, knowing the contraindications of Ticagrelor to prevent hemorrhagic complications. Case presentation: A 60-year-old man was admitted to. He had been receiving loading dose $180 \mathrm{mg}$ ticagrelor and $250 \mathrm{mg}$ aspirin and Heparin for a recent acute coronary syndrome (ACS) that had been treated with one baremetal stent and developed a major esophageal bleeding, pericardial effusion. 48 hours later, the patient had a decreased level of consciousness. Computed tomography (CT) revealed an intracranial hemorrhage peri-rolandic. Patient presented a cardiogenic shock (cardiac pump failure related to loss of myocardial contractility) with EF: 15\% (under dobutamine) and Hypovolemic shock (due to blood loss) combined. He proceeded to emergency cardiac transplantation operation. Apart from dual antiplatelet therapy (DAPT) with aspirin and ticagrelor, there were no other identified risk factors for increased bleeding. Conclusion: In cardiac surgical, patients who are treated with ticagrelor \& ASA until and after surgery, ticagrelor therapy is associated with a significantly higher blood loss, a significantly higher use of blood products and coagulation factors and higher incidence of rethoracotomies for bleeding. Consequently, Ticagrelor intake for ACS patients should be avoided or at least discontinued 3 days before cardiac surgery.
\end{abstract}

Keywords: Cardiac surgery, Ticagrelor, Hemorrhage, Corronary Artery Disease, ACS, Cardiogenic Shock, Hypovolemic shock

List of abbreviations

ACS(Acute coronary syndrome), CT (Computed tomography), ECG (Electrocardiograma),CAG (coronary angiography), CK (creatine kinase), ASA ( Acetylsalicylic Acid), STEMI (ST elevation myocardial infarction), NSTEMI (non ST elevation myocardial infarction), CAD (coronary artery disease), TV ( Tachycardia ventricular), DAPT (Dual Antiplatelet therapy), MI (myocardial infarction), CABG (Coronary artery bypass grafting )

\section{Introduction}

Ticagrelor is an orally active P2Y12 receptor blocker that does not require metabolic conversion to an active form. Ticagrelor binds directly to the P2Y12 receptor and can more completely inhibit the sustained platelet aggregation response to ADP than clopidogrel. The use of ticagrelor for reducing thrombotic cardiovascular events in patients with acute coronary syndromes is more suggested.

Ticagrelor is absorbed quickly from the gut, the bioavailability being $36 \%$, and reaches its peak concentration after about 1.5 hours. The main metabolite, AR-C124910XX, is formed quickly via CYP3A4 by dehydroxyethylation at position 5 of the cyclopentane ring. It peaks after about 2.5 hours. Both ticagrelor and ARC124910XX are bound to plasma proteins (>99.7\%), and both are pharmacologically active. Blood plasma concentrations are linearly dependent on the dose up to 1260 mg (the sevenfold daily dose). The metabolite reaches 30 $40 \%$ of ticagrelor's plasma concentrations. Drug and metabolite are mainly excreted via bile and feces.

Based on data from PLATO and TRITON-TIMI 38, the newer platelet inhibitors ticagrelor have been incorporated in the current European Society of Cardiology (ESC) guidelines[2]. Whilst their efficacy is undeniable, bleeding is an issue albeit at comparable rates with clopidogrel. We describe a case of internal bleeding, itself a rare entity, in a patient on ticagrelor post primary PCI for an leftmain STEMI, to underscore the risk of life threatening bleeding with the use of these new agents [3]

\section{Case Report}

A 59 years-old man, without the ACS risk factors, was admitted in Cardiologic Intensive Care Unit for an anterior ST-ACS in cardiogenic shock. A percutaneous coronary (PCI) was performed after a loading dose of unfractionated heparin (5000 UI), and found an occlusion of the left main coronary artery. Thrombo-ectomy and active stenting were performed while he received a loading dose of $250 \mathrm{mg}$ of aspirin and $180 \mathrm{mg}$ of ticagrelor. Secondary followed by a maintenance dose of respectively $75 \mathrm{mg}$ per day and $90 \mathrm{mg}$ twice a day.

Post-operative stay was complicated by a digestive hemorrhage and a major cardiac failure. Digestive endoscopy found a bleeding gastric ulcer, treated by local adrenalin injection and general daily injection of 


\section{International Journal of Science and Research (IJSR) \\ ISSN (Online): 2319-7064 \\ Index Copernicus Value (2013): 6.14 | Impact Factor (2015): 6.391}

esomeprazole. Concerning the cardiac failure, after a discussion with the heart team he was decided to register the patient on the national heart list transplant. In bridge to transplant, and Extracorporeal Life Support (ECLS) was implanted. HE underwent cardiac transplant 8 days later while on dual antiplatelet therapy (DAPT), using extracorporeal circulation with heparin (16 $000 \mathrm{UI})$, reversed by protamine (150 UI). Peri-operative blood loss was important because global hemorrhage without effect of protamine, cells transfusion (red cells, platelets), fibrinogen and plasma. Re-intervention happened a few hours later due to ongoing life threatening hemorrhage and disseminated intravascular coagulation (DIC): at opening, we found a mediastinal hematoma, a bilateral hemothorax around 2 liters and a cardiac tamponade. All cardiac stiches were blood free. Unfortunately, despite redo-surgery and polytransfusion, bleeding remains important and patient died on post-operative day one with a hemorrhagic shock and multi visceral organ failure. In total during the peri-operative course, he received 44 red cells units, 7 units of autologous platelet, 21 units of fresh frozen plasma, $12 \mathrm{mg}$ of proconvertine, and $10.5 \mathrm{mg}$ of fibrinogen.

\section{Discussion}

DAPT with aspirin and ticagrelor, there were no other identified bleeding in this patient?

Table 1: The variation of the blood panel

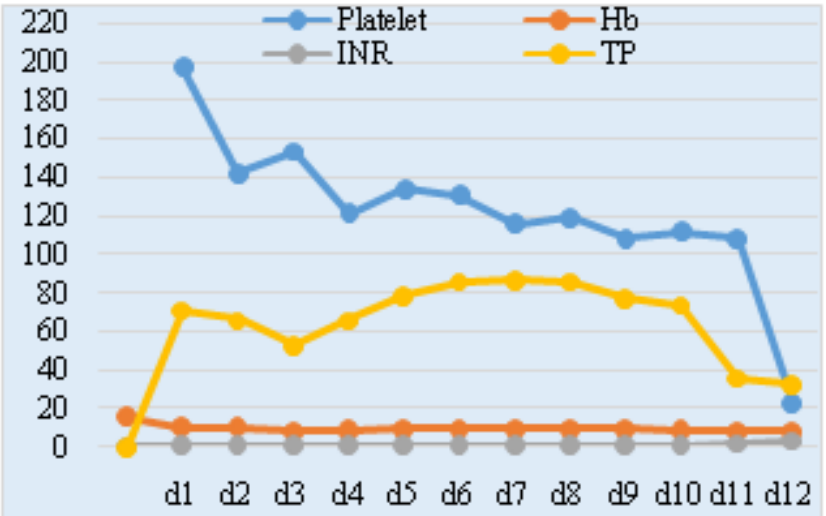

Blood panels showed a fall in haemoglobin from $15.8 \mathrm{~g} / \mathrm{dL}$ to $8.3 \mathrm{~g} / \mathrm{dL}$ with a fall platelet from 197 to 23 and at baseline his INR was 1.2. Ticagrelor is the first reversible oral ADP receptor antagonist available, exhibiting its antiplatelet effects by binding reversibly to platelet ADP P2Y12 receptors thus preventing its activation and aggregation. It has greater platelet inhibition compared to clopidogrel, with full functional recovery of circulating platelet activity as plasma levels fall. In the case presented, there were no identifiable factors (other than his DAPT) that predisposed the patient to bleeding.

\section{Bleeding risk of Ticagrelor? Study's Results}

Multiple studies with various baseline demographics using various bleeding definitions including Thrombolysis In Myocardial Infarction (TIMI), Bleeding Academic Research Consortium (BARC) and Platelet Inhibition and Patient Trial (PLATO) show different predisposing factors to bleeding.
A study 100 patients with Ticagrelor following PCI for STEACS $(n=45)$ or NSTEACS $(n=55)$ : At 30 days there was no death, fatal and minor bleeding (TIMI criteria). There were 19 (19\%) incidences of bleeding within the first 30 days. The majority of bleeding $(13 / 19,68 \%)$ was minimal bleeding from the access site. One patient (1\%) experienced major bleeding (colonic polyp) with a haemoglobin fall above $5 \mathrm{~g} / \mathrm{L}$. Five patients experienced bleeding requiring medical attention. Two of these patients had gastrointestinal bleeding (gastric ulcer, colon cancer), the rest presented with a haemoglobin drop without apparent primary source [4].

A nationwide study, all acute coronary syndrome patients in Sweden on dual antiplatelet therapy with aspirin and ticagrelor $(\mathrm{n}=1266)$ or clopidogrel $(\mathrm{n}=978)$ who underwent $\mathrm{CABG}$ during 2012-13 were included in a retrospective observational study. The incidence of major bleeding complications according to the Bleeding Academic Research Consortium-CABG definition was 38 and $31 \%$, respectively, when ticagrelor/clopidogrel was discontinued, $24 \mathrm{~h}$ before surgery. Within the ticagrelor group, there was no significant difference between discontinuation $72-120$ or $120 \mathrm{~h}$ before surgery (OR $0.93 ; 95 \%$ CI $0.53-1.64 ; \mathrm{P}=0.8$ ). In contrast, clopidogrel-treated patients had a higher incidence when discontinued $72-120$ vs $120 \mathrm{~h}$ before surgery (OR 1.71; 95\% CI $1.04-2.79 ; \mathrm{P}=0.033$ ). The overall incidence of major bleeding complications was lower with ticagrelor $(12.9 \%$ vs $17.6 \%$, adjusted OR $0.72 ; 95 \%$ CI $0.56-0.92 ; \mathrm{P}=0.012)$ [5].

\section{Inhibitory Effects of Ticagrelor}

This case highlights three important learning points. Firstly, despite the PLATO trial showing relative safety with ticagrelor there is a need for ongoing vigilance, especially given ticagrelor's greater platelet inhibition when compared to clopidogrel. Therefore, a high index of suspicion for bleeding in the setting of hemodynamic collapse whilst on these newer antiplatelet is important for the early diagnosis and treatment of potentially life threatening events. For patients who have the first clinically significant bleeding, we should discuss to decide whether antiplatelet therapy should be discontinued, for example in this patient: esophageal bleeding, intracranial hemorrhage... [6]

Secondly, for surgery with a high bleeding risk as cardiac surgery that requires the discontinuation of dual antiplatelet therapy, the strategy regarding the peri-procedural management of APA must be the result of a multidisciplinary consensus involving cardiologists or vascular specialists, anesthesiologists, and surgeons or interventional physicians. All non-urgent surgery should be delayed until the anti-P2Y12 drug can be safely withdrawn. In this case, if we need an emergency cardiac surgery for patients,ticagrelor should be stopped at least 24-96 hours before [7].

Finally, the antiplatelet of choice between clopidogrel and ticagrelor after stabilisation of a major bleeding episode in patients who are at risk of acute stent thrombosis is undefined. The decision for clopidogrel was made on the basis of our greater clinical experience with this drug and the fact that the patient had bled whilst on ticagrelor. To 


\section{International Journal of Science and Research (IJSR) \\ ISSN (Online): 2319-7064 \\ Index Copernicus Value (2013): 6.14 | Impact Factor (2015): 6.391}

compare hemorrhagic events between clopidogrel and ticagrelor in the Platelet Inhibition and Patient Outcomes (PLATO) trial, ticagrelor significantly increased spontaneous bleeds, major bleeds, major plus minor bleeds, and major plus minor plus minimal bleeds. Moreover, A study found that ticagrelor increased CABG-related bleeding versus clopidogrel in those patients who did not wait until day 5 after stopping treatment to have CABG. Additionally, compared to clopidogrel, ticagrelor increased the risk of hematuria (RR 1.91; 95\% CI 0.95-3.83), intracranial hemorrhage or subdural or other hematoma (RR 1.87; 95\% CI 1.02-3.42), subcutaneous hemorrhage, ecchymosis, hematoma (RR 1.63; 95\% CI 0.84-3.17), epistaxis (RR $1.49 ; 95 \%$ CI $0.67-3.32$ ), retroperitoneal hematoma or hemorrhage (RR 1.49; 95\% CI $0.53-4.19)$, gastrointestinal/anal bleed (RR 1.23; 95\% CI 0.93-1.64) and bleed/hematoma (RR 1.21; 95\% CI 1.02-1.43) [8].

In conclusion, it was specifically designed to address the limitations of the available antiplatelet agents while maintaining comparable or better antiplatelet effects. Nonetheless, ticagrelor is a drug that has the potential to change the standard of care of patients with acute coronary syndromes.

\section{References}

[1] Roffi M, Patrono C, Collet JP et al.2015 ESC Guidelines for the management of acute coronary syndromes in patients presenting without persistent STsegment elevation: Task Force for the Management of Acute Coronary Syndromes in Patients Presenting without Persistent ST-Segment Elevation of the European Society of Cardiology (ESC).Eur Heart J. 2016 Jan 14;37(3):267-315

[2] Amsterdam EA, Wenger NK, Brindis RG et al. 2014 AHA/ACC Guideline for the Management of Patients with Non-ST-Elevation Acute Coronary Syndromes: a report of the American College of Cardiology/American Heart Association Task Force on Practice Guidelines.J Am Coll Cardiol. 2014 Dec 23;64(24):e139-228

[3] Montalescot G, van't Hof AW, Lapostolle F et al. Prehospital Ticagrelor in ST-Segment Elevation Myocardial Infarction. N Engl J Med. 2014 Sep 11;371(11):1016-27

[4] Subiakto I, Asrar ul Haq M, Van Gaal WJ.Bleeding Risk and Incidence in Real World Percutaneous Coronary Intervention Patients with Ticagrelor. Heart Lung Circ. 2015 Apr;24(4):404-6

[5] Emma C. Hanssona, Helena Rexiusaet al. Coronary artery bypass grafting-related bleeding complications in real-life acute coronary syndrome patients treated with clopidogrel or ticagrelor. Eur J Cardiothorac Surg. 2014 Oct;46(4):699-705

[6] DiNicolantonio JJ et al. Clopidogrel is safer than ticagrelor in regard to bleeds: A closer look at the PLATO trial. Int J Cardiol. 2013 Oct 3;168(3):1739-44

[7] Bonhomme F, Fontana P, Reny JL.How to manage prasugrel and ticagrelor in daily practice. Eur J Intern Med. 2014 Mar;25(3):213-20

[8] Held C et al. Ticagrelor Versus Clopidogrel in Patients With Acute Coronary Syndromes Undergoing Coronary Artery Bypass Surgery - Results From the PLATO
(Platelet Inhibition and Patient Outcomes) Trial. J Am

Coll Cardiol. 2011 Feb 8;57(6):672-84 